\title{
Discussion A: On the abundance of deuterium in the local interstellar medium and in high-redshift systems
}

\author{
Monica Tosi \\ INAF - Osservatorio Astronomico di Bologna \\ Via Ranzani 1, I-40127, Bologna, Italy \\ email: monica.tosi@oabo.inaf.it
}

\begin{abstract}
The first discussion session held at the IAU Symposium 268 focussed on the deuterium content in the local interstellar medium (LISM) and in high-redshift systems. There were two key questions proposed to the audience: 1) what should be taken as representative abundance of D in the LISM, and 2) how can we explain the dispersion of the D abundance measured in high-redshift, very low metallicity environments? While on the latter point people seem to agree that observational and data analyses uncertainties are the most likely explanation, on the former question no consensus was reached. The historical and observational background at the basis of these questions and the discussion are schematically reported here.
\end{abstract}

Keywords. ISM: abundances; Galaxy: evolution; cosmology: observations; quasars: absorption lines

\section{Introduction}

Four discussion sessions were held at the IAU Symposium 268 on the most debated issues related to the light elements. The first of these special sessions was devoted to our current understanding of the deuterium abundance in the local interstellar medium (LISM) and in high-redshift systems. Two key questions were selected by the SOC as the current hottest topics on deuterium and opened to discussion: 1) what should be taken as representative abundance of D in the LISM, and 2) how can we explain the dispersion of the D abundance measured in high-redshift, very low metallicity environments. In the recent literature there have been quite interesting debates on both instances and the lively discussion which took place at the meeting reflected the deep involvement of the community.

The very circumstance that these questions need to be asked is the positive result of the efforts and achievements of scientists exploiting modern, high-performance instruments, such as ground-based high-resolution spectrographs at $10 \mathrm{~m}$ class telescopes and those on the HST and FUSE satellites. On the high-redshift side, the data currently available on D have been summarized in the recent papers by O'Meara et al. (2006) and Pettini et al. (2008). On the Galactic side, Geiss, Hébrard, Linsky and Sembach have comprehensively described here (this volume) the available measurements. From their presentations, it is apparent that the increasing number of accurate measures has led to a much larger coverage of different environments. As summarized by Savage et al. (2007), we now have D measured in the solar system, in the LISM, in a couple of fields in the Galactic disk and halo, in a high-velocity cloud (Complex C) and in several Damped Lyman- $\alpha$ systems (DLAs). Yet, we have reached neither a clear understanding of the deuterium distribution in different environments, nor a consensus on its most representative values. 


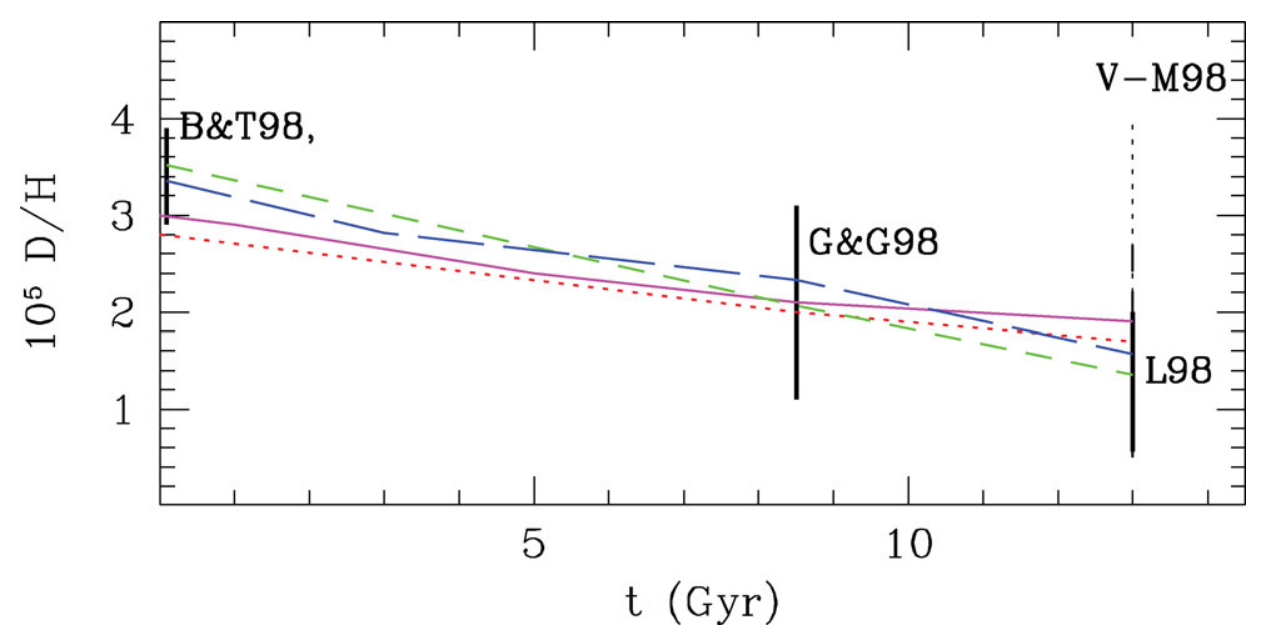

Figure 1. What we knew of the deuterium abundances by number ten years ago (see Tosi 2000). The coloured curves show the abundance variation with time as predicted by chemical evolution models for the solar neighbourhood. These curves refer to models by different groups, all able to reproduce the majority of the Galaxy observational constraints (see for references Tosi 2000). The vertical bars show at $2 \sigma$ the abundances estimated by Burles \& Tytler (1998) for high redshift absorbers, by Geiss \& Gloeckler (1998) for the pre-solar cloud and by Linsky (1998) (solid bar) and Vidal-Madjar et al. (1998) (dashed and dotted bars) for the LISM.

Ten years ago the situation looked much simpler and stable. At the IAU Symposium 198 on the Light Elements, held in Natal (Brasil) in 1999, the main players in the D measurement game showed $\mathrm{D} / \mathrm{H}$ values with rather small dispersions, almost undistinguishable from those presented two years earlier at the meeting on the same topic organized by the International Space Science Institute (ISSI) in Bern (Switzerland). These values are schematically plotted in Fig. 1 (showed at the IAU Symp.198) and display a steady and moderate decrease from the close-to-primordial $\mathrm{D} / \mathrm{H}$ of high-z absorbers (Burles \& Tytler 1998), to the proto-solar cloud (PSC) value inferred from solar-system data (Geiss \& Gloeckler 1998), to the abundance (Linsky 1998) in the Local Interstellar Cloud (LIC). Assuming the high-z values as typical of proto-galactic clouds 13 Gyr ago, the PSC value as typical of the LISM at the time of the Sun formation 4.5 Gyr ago, and the LIC value as typical of the LISM at the present epoch, the plotted trend traces the evolution of $\mathrm{D}$ in the solar neighbourhood during the Galaxy lifetime.

Ten years ago, Tytler and collaborators had just demonstrated that the cases of high-z absorbers where $\mathrm{D} / \mathrm{H}$ ratios almost an order of magnitude higher had been claimed to exist (e.g. Songaila et al. 1994) were actually misinterpretations of interloopers or of the continuum level in the observed spectra. Chemical evolution models able to reproduce the vast majority of the Galaxy observed properties were nicely consistent with these $\mathrm{D} / \mathrm{H}$ data (coloured lines in Fig. 1), including the value 9 times lower than the local ones measured by Lubowich et al. (2000) in the Galactic center, $(\mathrm{D} / \mathrm{H})_{G C}=1.7 \pm 0.3 \mathrm{ppm}$. People felt reassured and satisfied.

There were actually voices of warning (Vidal-Madjar et al. 1998), arguing that D/H in the LISM appeared to vary significantly from one line-of-sight (LOS) to the other, but the establishment tended to disclaim those arguments, although admitting that the constant values were actually confined within the quite small region (100 pc radius) of the Local Bubble (LB). The dashed and dotted vertical bars in Fig. 1 show respectively 
the likely range of LISM values and the less likely one including the maximum, possibly wrong, $\mathrm{D} / \mathrm{H}=4 \mathrm{ppm}$ ever estimated (see Vidal-Madjar et al. 1998 for references).

\section{What do we currently know of the local D abundance?}

In ancient Greece mythology Cassandra's profecies were never believed either by the Trojans or by the Achaeans, but she was always right. So were the skeptics about the homogeneity of LISM deuterium. If we look at the distribution of $\mathrm{D} / \mathrm{H}$ with column density $\mathrm{N}(\mathrm{HI})$ (which can be considered a proxy for distance), as resulting now from years of analyses of both old and new data (see Fig. 2, taken from Linsky et al. 2006), it is apparent that $\mathrm{D} / \mathrm{H}$ varies significantly. Only the data within the $\mathrm{LB}$ are tight to the $\mathrm{D} / \mathrm{H}$ value which was attributed to the LISM ten years ago. Regions with $\log \mathrm{N}(\mathrm{HI}) \geqslant$ 20.7 seem to have much lower $\mathrm{D} / \mathrm{H}$ (lower $\mathrm{D}$ or higher $\mathrm{H}$ ?) and regions with intermediate column densities show an impressive spread, which is now convincingly explained in terms of spatially varying D depletion onto dust grains (Jura 1982, Draine 2004, Linsky et al. 2006). So, what value, if any, could actually be taken as the current "average" LISM deuterium? Is it the upper undepleted value $23 \pm 2.4 \mathrm{ppm}$ of the intermediate region, or a lower value (say $20 \pm 1 \mathrm{ppm}$ ) allowing for observational errors, or the very low value $9.8 \pm 1.9 \mathrm{ppm}$ of the highest column density regions? Different authors (Linsky et al. 2006, Prodanovich et al. 2009, Hébrard et al. 2005, respectively) have suggested each of these possibilities and the question is still open.

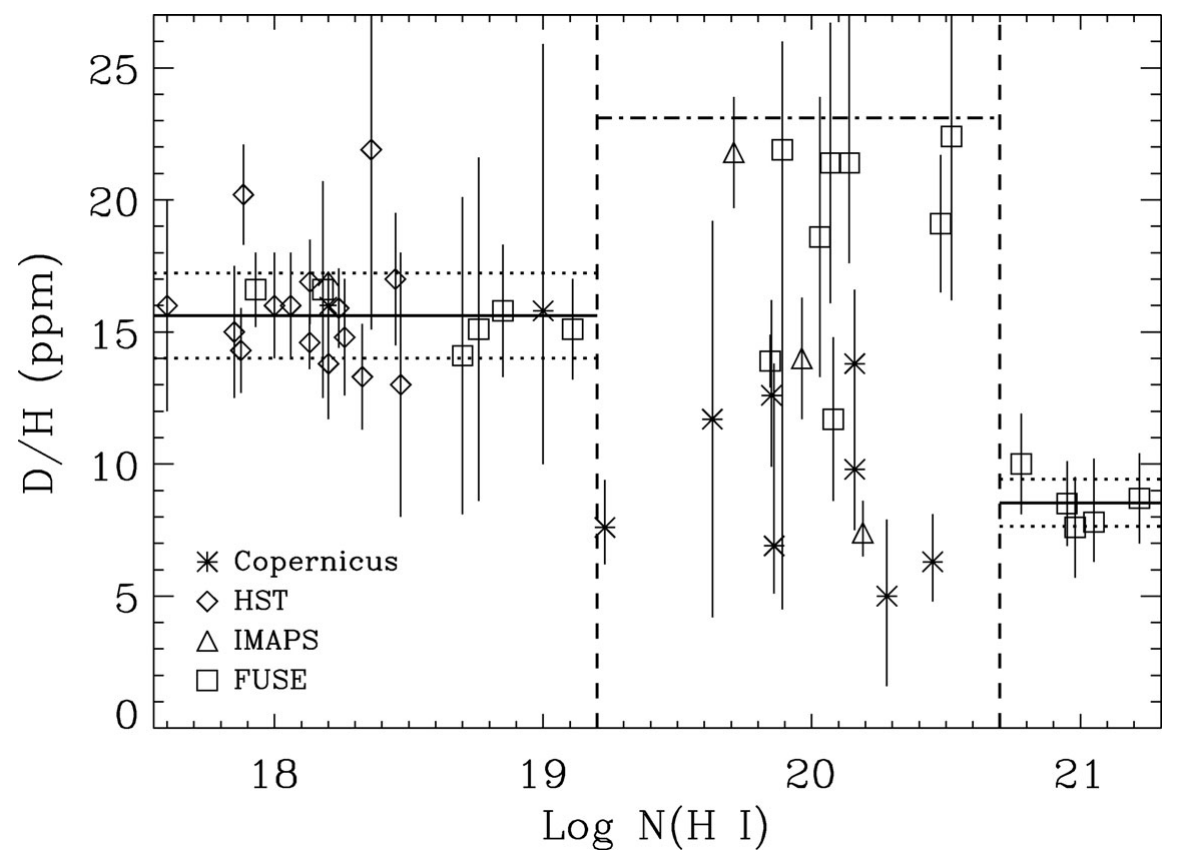

Figure 2. From Linsky et al. (2006): deuterium abundance vs hydrogen column density measured along several LOS in the LISM.

Each of these possibilities also has side effects on our understanding of the chemical evolution of the Galaxy. If the LISM D/H is as high as $23 \pm 2.4 \mathrm{ppm}$, at face value it is higher than in the PSC (21 \pm 5 ppm, Geiss \& Gloeckler 1998) and implies either a way to enhance D in the last $4.5 \mathrm{Gyr}$ (quite unlikely) or the need to consider the Sun not representative of the local medium at the time of its formation (a fairly recurrent 
theme, never settled with satisfactory arguments). Moreover, if the primordial $\mathrm{D} / \mathrm{H}$ is $(\mathrm{D} / \mathrm{H})_{P}=26.1 \pm 3 \mathrm{ppm}$ as implied by the first modelling of the WMAP data (Spergel et al. 2003) the total deuterium astration factor from the Big Bang to a present LISM $\mathrm{D} / \mathrm{H}=23 \mathrm{ppm}$ would be 1.13 , quite low even for chemical evolution models allowing for the continuous accretion of large amounts of primordial extragalactic gas, but still consistent with the Galaxy properties (Steigman et al. 2007). Once we consider that the infalling gas is probably not primordial and more likely with a $\mathrm{D}$ content similar to that of high velocity clouds (Sembach et al. 2004 estimated a $\mathrm{D} / \mathrm{H}=22 \pm 7 \mathrm{ppm}$ in Complex $\mathrm{C})$, it becomes clear that no viable model can account for such values. If $(\mathrm{D} / \mathrm{H})_{P}=28.2$ as recently suggested by Pettini et al. (2008), the astration factor to $\mathrm{D} / \mathrm{H}=23 \mathrm{ppm}$ would be 1.22 , still challenging, but not impossible to achieve. Models consistent with the various Galactic constraints predict astration factors larger than 1.3 (e.g. Romano et al. 2006, Steigman et al. 2007) and can reproduce a LISM D/H of 19-20 ppm or lower with standard assumptions on metal poor infall. Most likely one should consider spatial variations of depleting dust grains and of the accreted metal-poor gas to account for the empirical inhomogeneity of the D abundances.

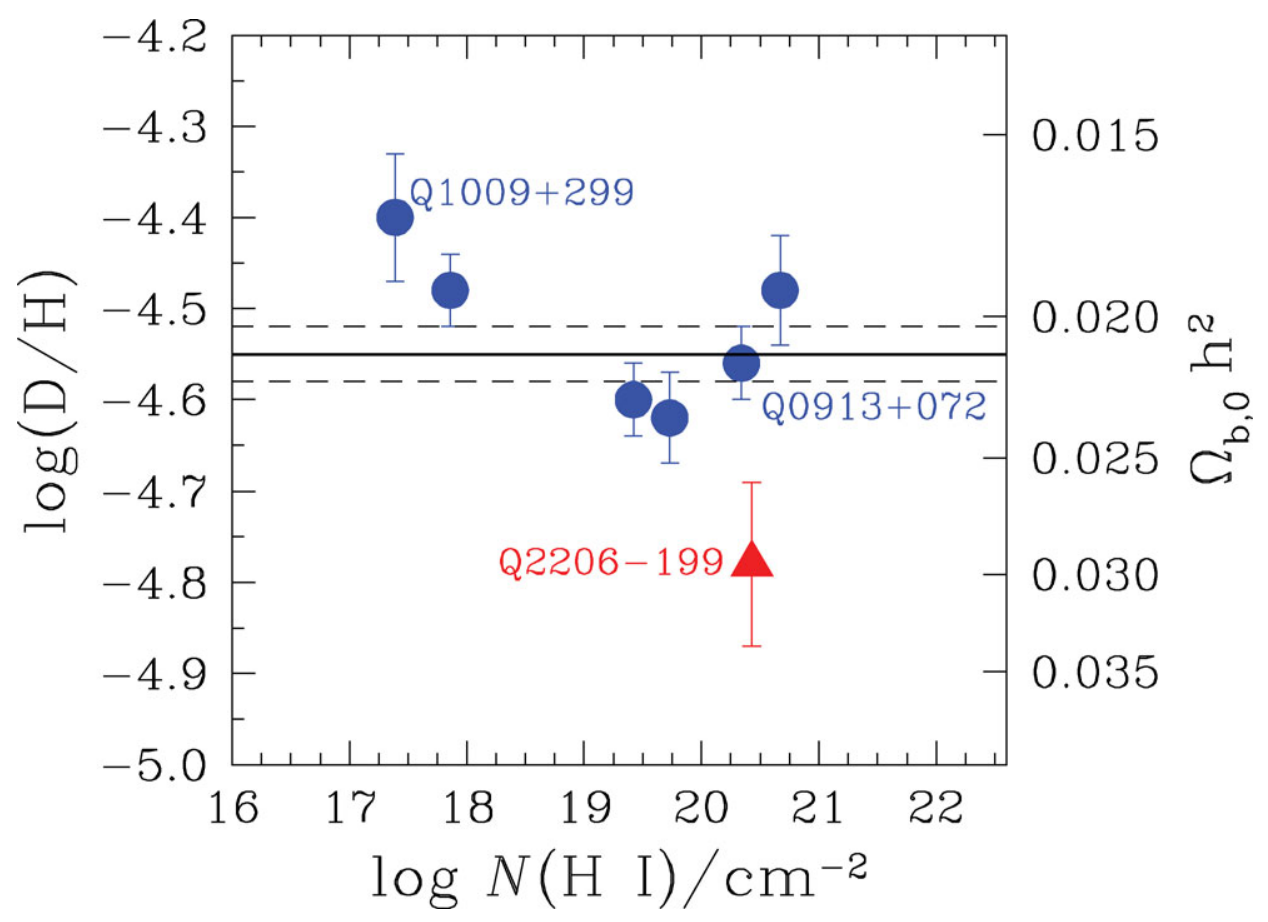

Figure 3. From Pettini et al. (2008): The deuterium abundance measured in high-redshift DLAs. The horizontal lines show their mean value (solid) $\pm 1 \sigma$ (dashed). On this scale the primordial D inferred from WMAP is -4.59 .

\section{What do we currently understand of high-redshift deuterium?}

Outside our Galaxy deuterium is measured in the absorption lines of gas systems falling on the LOS of QSOs with redshift lower than 4. Fig. 3 (taken from Pettini et al. 2008) illustrates these data and the corresponding mean deuterium value, 28.2 ppm. All measurements are consistent at $1 \sigma$ with this mean, except two values, which happen however to be the most uncertain ones, one because of the lower $\mathrm{S} / \mathrm{N}$ of its 
spectra (the red triangle in Fig. 3) and the other because D is detected only in the Ly- $\alpha$ line.

Should this mean value of $28.2 \mathrm{ppm}$ be taken as the best estimate of the primordial $\mathrm{D} / \mathrm{H}$ ? At face value, it is slightly larger than - although consistent with - the values $(\mathrm{D} / \mathrm{H})_{P}=26.1 \pm 3 \mathrm{ppm}$, or $(\mathrm{D} / \mathrm{H})_{P}=25.7 \pm 1.5 \mathrm{ppm}$ estimated from WMAP after the first year and the third year data release (Spergel et al. 2003 and Spergel et al. 2007, respectively). If real, this difference would be unexplainable, because DLAs have very low but non zero metals. Since metals are produced by stars, and stars always destroy D, DLAs are supposed to have D somewhat lower than primordial. If the DLA measurements can be considered sufficiently robust, Pettini's heuristical and backward approach of using the observed high-z $\mathrm{D} / \mathrm{H}$ as a prior in the analysis of WMAP data appears very reasonable. Can we follow his arguments and conclude that $(\mathrm{D} / \mathrm{H})_{P}$ is more likely $28.2 \mathrm{ppm} ?$

If we consider both the deuterium and oxygen abundances of these systems, we find that DLAs with higher O may also have higher D, contrary to basic nucleosynthesis principles. Is this also due to observational issues or should we worry? And, more generally, are the apparent differences in $\mathrm{D} / \mathrm{H}$ from one absorption system to the other real? If so, what is the physical meaning of the high-z values different from the average value?

\section{Discussion}

First of all, during the discussion session the participants, sollicited by Ken Sembach and me, agreed that one cannot assume the LISM D value as representative of the value for the Galaxy as a whole, or even for the local spiral arm. It is not clear whether we can actually identify a mean LISM value, but it is evident that such value could not be representative of other Galactic regions with different conditions and evolution.

The quest for the actual D abundances here and now (LISM), and there and then (high-z absorbers) was then debated at length.

Jeff Linsky noticed that in many papers authors implicitly assume that a measurement of $\mathrm{D} / \mathrm{H}$ in the gas phase is a measure of the total $\mathrm{D} / \mathrm{H}$ ratio, and urged authors to make a clear distinction between the gas phase $\mathrm{D} / \mathrm{H}$ and the total $\mathrm{D} / \mathrm{H}$ that include $\mathrm{D}$ in dust grains. Following up on this point, Donatella Romano asked if, on the other hand, there could be undetected molecular hydrogen along some FUSE LOS. If so, could the contribution from this unaccounted $\mathrm{H}_{2}$ help to bring the highest observed values of $\mathrm{D} / \mathrm{H}$ in the local ISM in agreement with the predictions on D evolution from standard chemical evolution models? Linsky answered that all $\mathrm{H}_{2}$ was included in the estimate of $\log \mathrm{N}(\mathrm{H})$ and that, in any case, it provides only a negligible fraction of the total $\mathrm{H}$ column density.

Gary Steigman described in some detail his and Tijana Prodanovic' attempts on using both D and Fe to try to find the "true" (i.e., gas plus dust) ISM D abundance. Based on $\log \left(y_{D}\right)$ vs. $\log \left(y_{F e}\right)$ plots $\left[\right.$ where $y_{D}=10^{5}(\mathrm{D} / \mathrm{H})$ and $\left.y_{F e}=10^{6}(\mathrm{Fe} / \mathrm{H})\right]$, he argued that the LB D abundance shows no evidence for any variation, in contrast to the $\mathrm{LB} F$ abundance, suggesting that D may not be depleted in there (i.e., no correlation between $\mathrm{D}$ and $\mathrm{Fe}$ ). Most of the non-LB LOS have lower Fe abundances than in the LB, and for most of them there is an apparent correlation between D and Fe. However, there are 6 remaining non-LB LOS which have D abundances in excess of the LB value, and he suggested that these high D and (mostly) low Fe abundances might have resulted from incompletely mixed infall, so that the "true" ISM D abundance might be the LB value $y_{D, L B}=1.5$, corresponding to a $\mathrm{D}$ astration factor of 1.8. An alternative interpretation of the data is that the "true" ISM D abundance is the value $\left(y_{D, I S M}=2.0\right)$ found by Prodanovic et al. (2009) from Bayesian analysis. The potential problem with this choice 
is that the LB D abundance is uniform but depleted compared to the ISM value by a factor of 2.0/1.5 =1.3. In response to this speculation that the high $\mathrm{D}$ abundances might be due to infall, Guillaume Hébrard remarked however that the oxygen abundance along these LOS is "normal", and not low as would be expected for metal-poor gas.

Sembach asked what future observations of D or other species might help resolve the issue of deuterium depletion onto dust, but no encouraging answer was provided by the audience, either in terms of currently usable instrumentation or of tracing D through other elements. He himself did not see many future prospects for FUV DI absorption measures for gas within the Milky Way for two reasons. First, there is no obvious followon to FUSE in NASA's strategic plan (HST/COS could make some breakthroughs in the low-reshift IGM arena, however, with very interesting output). Second, within the Milky Way these types of measurements are confusion limited. Thus, it may be that nature simply doesn't provide a simple enough velocity structure along the vast majority of extended sight lines to be able to infer $\mathrm{D} / \mathrm{H}$ or $\mathrm{D} / \mathrm{O}$ much beyond a kiloparsec from the Sun. Higher resolution $(\mathrm{R} \geqslant 50,000)$ observations might help in understanding this limitation better and may help push the distance envelope a factor of two or three.

Tom Bania replied reporting on the first solid detection of the D hyperfine transition at $327 \mathrm{MHz}$ in the Milky Way ISM (Rogers et al. 2005, Rogers et al. 2007). Unfortunately, the array is now dismissed and there are no plans to either repeat or extend these measurements. In contrast to all other $\mathrm{D} / \mathrm{H}$ abundance determinations these measurements are not made toward specific targets but rather probe specific Galactic directions. The three fields are all in the Galactic anticenter direction and latitude $0 \mathrm{deg}$. The average $\mathrm{D} / \mathrm{H}$ abundance derived for these three fields is $\langle\mathrm{D} / \mathrm{H}\rangle=21 \pm 7 \mathrm{ppm}$, where the error is $\pm 3 \sigma$ and contains an estimate for the uncertainty in the HI excitation temperature. In one direction, $\mathrm{D} / \mathrm{H}=24 \mathrm{ppm}$ is found at a SNR of 8.2 with a total integration time of 17.5 years. No one is going to supersede these $92 \mathrm{~cm}$ measurements any time soon. Bania and coworkers estimate that $\mathrm{D}$ is distributed over $\sim 5 \mathrm{kpc}$ in these Galactic anticenter directions, with no significant difference between the three. This makes this path length as long as any of the measured optical paths for D LOS in the Milky Way. For this $\sim 5 \mathrm{kpc}$ path the HI column density is $3 \times 10^{21} \mathrm{~cm}^{-2}$. Sembach commented that, while the FUSE results apply over a very limited range of distances, the measures of the DI $92 \mathrm{~cm}$ emission might have more applicability in defining an "average" Galactic value, since those types of measurements can potentially sample large volumes of the Galaxy.

With reference to the two questions raised before, (1) how much molecular hydrogen is likely to be found in these LOS, and (2) is this a gas phase measurement only, Bania argued that each of these LOS probes DI emission from an enormous volume of the ISM, unlike the pencil beams absorbing the continuum radiation from a background target object. At a distance of $5 \mathrm{kpc}$ the beam extends to $\pm 610 \mathrm{pc}$ from the Galactic plane. This is $\sim 15$ times the 40 pc scale-height of the molecular gas disk of the Milky Way. Thus most of the volume probed by the DI measurements should be devoid of molecular gas. There should be, on average, very little molecular hydrogen contamination on the corresponding $\mathrm{D} / \mathrm{H}$ abundance derivation. The possibility that significant amounts of $\mathrm{D}$ has frozen out onto grains cannot be discounted, but Bania believes that their LOS may actually be the cleanest available in this regard, because most of their probed lies far above the dense, cold material in the Galactic plane. Most of their observed volume contains warm neutral medium (WNM) gas at temperatures between 1,000 and 4,000 K. Grains mixed into this environment are unlikely to be cold enough to freeze out D. Hence, the fraction of observed volume wherein D can freeze out onto grains is too small to significanly skew their $\mathrm{D} / \mathrm{H}$ abundance determination. 
Given the uncertainties in accurately deriving D/H, Guillaume Hébrard argued that a comparison of $\mathrm{D}$ with $\mathrm{O}$ may be more reliable. He pointed out that the $\mathrm{D} / \mathrm{O}$ ratio appears much more homogeneous than $\mathrm{D} / \mathrm{H}$. He thus suggested that the distant low $\mathrm{D} / \mathrm{O}$ and $\mathrm{D} / \mathrm{H}$ ratios are more likely representative of the local current values, whereas the high $\mathrm{D} / \mathrm{H}$ for which no high $\mathrm{D} / \mathrm{O}$ are measured could be due to systematics. Linsky agreed that it is important to estimate $\mathrm{D} / \mathrm{H}$ by different techniques, but believes that estimating $\mathrm{D} / \mathrm{H}$ through another element like oxygen $(\mathrm{D} / \mathrm{H}=\mathrm{D} / \mathrm{O} \times \mathrm{O} / \mathrm{H})$ introduces a whole new set of poorly understood problems in addition to the problems in understanding $\mathrm{D} / \mathrm{H}$. One needs to know the ionization equilibrium, depletion, and perhaps also the distribution of the element along the LOS which will be different than for D and H. Also, chemical evolution, depletion, and mixing could be different for this element than for $\mathrm{H}$ and D.

Chris Howk mentioned that for Galactic measurements, there is almost certainly some bias due to the manner in which sight lines were chosen for analysis from the FUSE database. Given the work required for each individual sight line, there is a tendency to choose sight lines with visible deuterium absorption. This means the DI is separated from the HI absorption, but it potentially also biases the results against low D sight lines. How large a bias this represents is unknown. Sembach confirmed that the sight lines sampled by FUSE for both $\mathrm{D} / \mathrm{H}$ and $\mathrm{D} / \mathrm{O}$ measurements have strong selection effects. These are kinematically simple sight lines that probe the warm diffuse ISM in which there is little molecular hydrogen. Dust grains in these types of regions show no evidence of icy mantles. These types of regions tend to have mantles that are highly processed by shocks. These regions are not representative of the types of regions where grains mantles are built. Rather, they are regions grains inhabit after they leave darker cloud environments. He wondered if anyone has done (or will do) a systematic assessment of the FUSE archive to determine if there are any sight lines for which DI absorption could have been detected but wasn't. In other words, are there any "D-free" sight lines that might be telling us something interesting? Nobody seemed able to answer this question.

Sembach also pointed out that a correlation between the inferred depletion of deuterium and heavier elements such as $\mathrm{Fe}$ and $\mathrm{Ti}$ is not surprising, but one should be puzzled by how little variation there is about the trend. Given that Fe or Ti are depleted by factors of 100-1000, compared to at most a factor of 2 for D, even a small change in the mantle properties should produce a large change in the gas-phase abundance of $\mathrm{Fe}$ or Ti. In other words, one could double the gas-phase abundance of Ti or Fe easily by releasing only 1 part in 100 or 1000 of the Fe or Ti back into the gas, whereas a doubling of the $\mathrm{D}$ abundance would require essentially all of the $\mathrm{D}$ to be released back into the gas phase. The observed data seem at odds with there being a simple distribution of $\mathrm{D}$ and the heavier elements within dust grains if this is indeed the explanation for the variations in the $\mathrm{D} / \mathrm{H}$ ratio. These arguments made him skeptical, as they suggest there could still be considerable unknown (systematic) uncertainties associated with the values of the HI column densities along some of these sight lines.

Finally, Sembach recalled that, although difficult to test observationally, some of the LOS to LOS variation in $\mathrm{D} / \mathrm{H}$ could be due to local sources of the type discussed during the conference. These sources are insufficient to produce cosmological quantities of D, but he saw no particular reason they couldn't add to the variation in deuterium abundances seen in different directions. The importance of such sources remains indeed to be determined.

To summarize, it is apparent that there is no consensus on which is the actual deuterium abundance of the LISM, or even on whether a representative value can exist. Clearly further studies are necessary to better understand this issue, but adequate instruments are currently missing. As pointed out by Linsky, we need a new spectrograph 
for the 912-1200 A spectral range, with higher sensitivity and spectral resolution than FUSE. This would allow a deeper insight on the local abundance distribution as well as $\mathrm{D} / \mathrm{H}$ measurements beyond $1 \mathrm{kpc}$ providing information on the dependence of $\mathrm{D} / \mathrm{H}$ with radial position in the disk and halo of the Galaxy and in neighboring galaxies like the Magellenic Clouds. Radio arrays able to measure the ground-state spin-flip transition of $\mathrm{D}$ at $327 \mathrm{MHz}(92 \mathrm{~cm})$ in several Galactic longitudes, to add to those described by Rogers et al. (2007) could be useful too.

Concerning high-z absorbers, Joanna Dunkley argued that, when comparing results for the deuterium abundance from WMAP and the Pettini et al. (2008) measurements, it is worth noting that there is a less than $2 \sigma$ difference between the two. Since they are statistically consistent, at this stage it may not be worth investing much time in choosing one or the other. However, to try to compare the two measurements, one should consider how robust the WMAP result is. WMAP has very low systematics, leading to a precise measurement of the angular power spectrum. Inferring the baryon density from the power spectrum also relies on linear physics that we understand. We can numerically compute the expected spectrum for a given cosmological model to high precision, so the estimated density should not include additional uncertainties. There is a small dependence of the estimated baryon density on the cosmological model assumed; for example, the estimate moves by about $1 \sigma$ if we extend the standard $\Lambda$ CDM cosmological model to marginalize over a running spectral index, or a varying equation of state of dark energy (Komatsu et al. 2009). However, there is currently no evidence that these extended models are favored over $\Lambda$ CDM. The final step, i.e. inferring the $\mathrm{D}$ abundance from the baryon density, assumes the standard Big Bang model. While this is consistent with the CMB observations, there could be extensions to this model to be invoked to explain the apparent discrepancy in the lithium abundance (discussed in Jedamzik's talk, this volume), that could modify this inference.

Finally, Paolo Molaro remarked that after almost two decades of $10 \mathrm{~m}$ telescope efforts we remain with only 8 measurements. Moreover, these measurements show a dispersion which exceeds the reported errors, thus suggesting either the presence of a scatter in the $\mathrm{D} / \mathrm{H}$ or an underestimate of the errors. He recalled two aspects to consider. First, the measurements are obtained towards absorbers with neutral hydrogen column densities differing by more than two orders of magnitude, which normally are referred to different classes of objects. Given that the major source of error is the estimate of the hydrogen column density, he would regard the determination obtained towards damped wings of the hydrogen lines in the DLAs somewhat more reliable than in the other systems. A second aspect is that these systems show low metallicities but within a quite large range $-3 \leqslant[\mathrm{Si} / \mathrm{H}] \leqslant-1$. At these metallicities he believes that no significant astration can have occurred and there is no hint of correlation between $\mathrm{D} / \mathrm{H}$ and metallicities. However, considering that the measurements in the Galaxy show a correlation with the Fe abundances, suggesting a depletion of D into dust grains, it would be important to check if any correlation exists between the $\mathrm{D} / \mathrm{H}$ abundance and the gas depletion factor. Unfortunately, a robust estimate of depletion can be obtained only from $\mathrm{Zn}$ abundances, which are not available for the observed systems: certainly a check to be done in more detail in future observations.

\section{Acknowledgements}

The Swiss National Science Foundation and IAU are gratefully acknowledged for financial support. Partial support comes also from the Italian PRIN-MIUR-2007. I'm grateful to Max Pettini for useful and updated information, and to Tom Bania, Joanna Dunkley, Guillaume Hébrard, Chris Howk, Jeff Linsky, Paolo Molaro, Donatella Romano, Ken 
Sembach, and Gary Steigman for their active participation to the discussion and for their contribution to this report. Fruitful and pleasant meetings and discussions on this topic held at ISSI in Bern are also acknowledged.

\section{References}

Burles, S. \& Tytler, D. 1998, in Primordial Nuclei and their Galactic evolution, N. Prantzos, M. Tosi, R. von Steiger eds., Space Science Reviews 84, 65

Draine, B. T. 2004, in Origin and evolution of the elements, A. McWilliams, M. Rauch eds, CUP, p.317

Geiss, J. \& Gloeckler, G. 1998, in Primordial Nuclei and their Galactic evolution, N. Prantzos, M. Tosi, R. von Steiger eds., Space Science Reviews 84, 239

Hébrard, G., Tripp, T. M., Chayer, P., Friedman, S. D., Dupuis, J., Sonnetrucker, P., Williger, G. M., \& Moos, H. W. 2005, ApJ, 65, 1136

Jura, M. 1982, in Advances in UV astronomy, Y. Kondo, J. Mead, R. D. Chapman eds., NASA, p. 54

Komatsu, E., Dunkley, J. et al. 2009, ApJS, 180, 330

Linsky, J. L. 1998, in Primordial Nuclei and their Galactic evolution, N. Prantzos, M. Tosi, R. von Steiger eds., Space Science Reviews 84, 285

Linsky, J. L., Draine, B. T., Moos, H. W., Jenkins, E. B. et al. 2006, ApJ 647, 1106

Lubowich, D. A., Pasachoff, J. M., Balonek, T. J., Millar, T. J., Tremonti, C., Roberts, H., \& Galloway, R. P. 2000, Nature, 405, 1025

O’Meara, J. M., Burles, S., Prochaska, J. X., Prochter, G. E., Bernstein, R. A., \& Burgess, K. M. 2006 ApJ, 649, L61

Pettini, M., Zych, B. J., Murphy, M. T., Lewis, A., \& Steidel, C. C. 2008, MNRAS, 391, 1499

Prodanovic, T., Steigman, G., \& Fields, B. D. 2009, arXiv:0910.4961

Rogers, A. E. E., Dudevoir, K. A., Carter, J. C., Fanous, B. J., Kratzenberg, E., \& Bania, T. M. 2005, ApJ, 630, L41

Rogers, A. E. E., Dudevoir, K. A., \& Bania, T. M. 2007, AJ, 133, 1625

Romano, D., Tosi, M., Chiappini, C., \& Matteucci, F. 2006, MNRAS, 369, 295

Savage, B. D., Lehner, N., Fox, A., Wakker, B., \& Sembach, K. 2007, ApJ, 659, 1222

Sembach, K. et al. 2004, ApJS, 150, 387

Songaila, A., Cowie, L. L., Hogan, C. J., \& Rugers, M. 1994, Nature, 368, 599

Spergel, D. N. et al. 2003, ApJS, 148, 175

Spergel, D. N. et al. 2007, ApJS, 170, 377

Steigman, G., Romano, D., \& Tosi, M. 2007, MNRAS, 378, 576

Tosi, M. 2000, in The light elements and their evolution, IAU Symp.198, ASP Conf.Ser., L. DaSilva, M. Spite, J. R. do Medeiros eds., p. 525

Vidal-Madjar, A., Ferlet, R., \& Lemoine, M. 1998, in Primordial Nuclei and their Galactic evolution, N. Prantzos, M. Tosi, R. von Steiger eds., Space Science Reviews 84, 297 


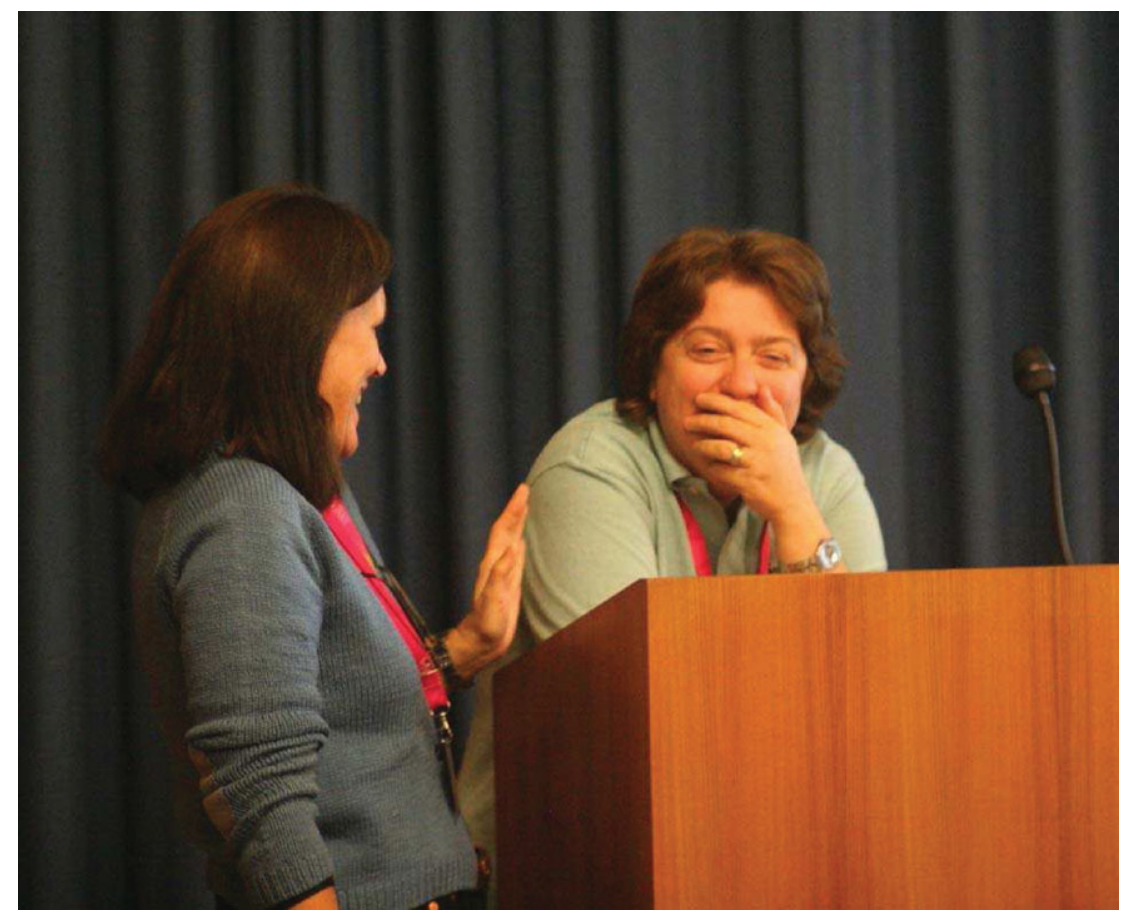

Monica Tosi \& Angela Bragaglia

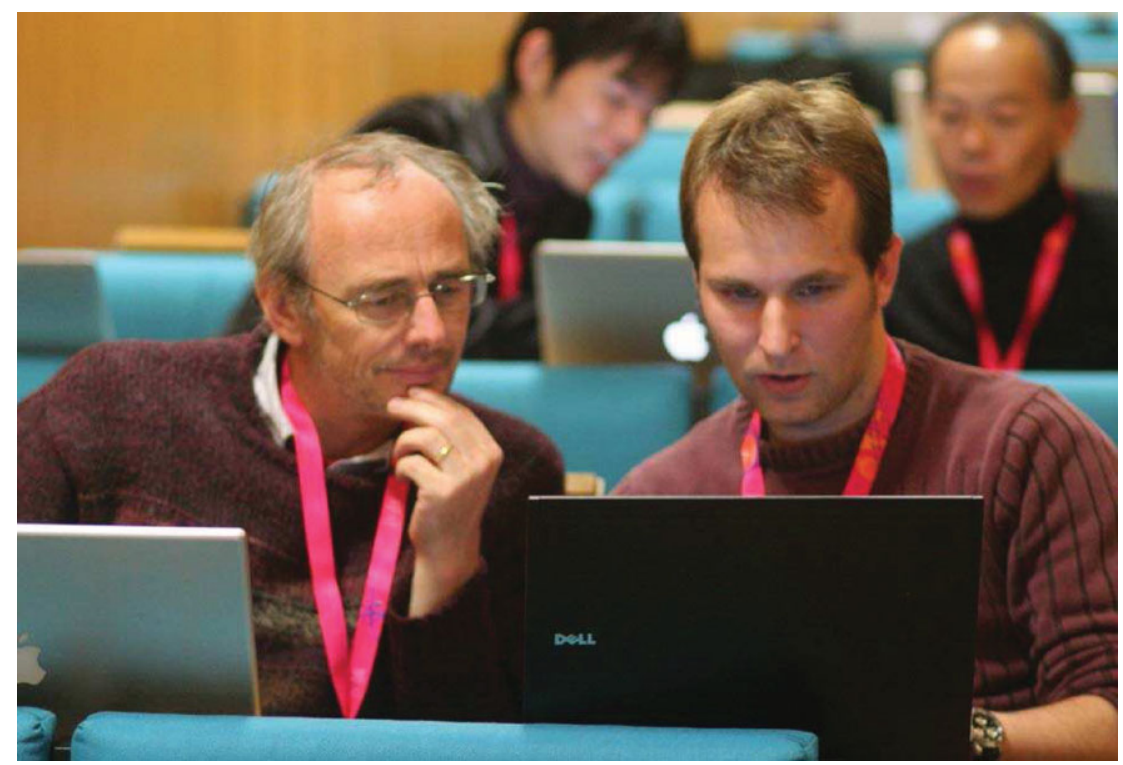

Georges Meynet \& Urs Frischknecht 